\title{
Generation of cortical neurons through large-scale expanding neuroepithelial stem cell from human pluripotent stem cells
}

\author{
Shumei Zhao ${ }^{1+}$, Kui Duan ${ }^{1+}$, Zongyong Ai ${ }^{1}$, Baohua Niu', Yanying Chen ${ }^{1}$, Ruize Kong ${ }^{1}$ and Tianqing $\mathrm{Li}^{1,2^{*}}$
}

\begin{abstract}
Background: Considerable progress has been made in converting human pluripotent stem cells (hPSCs) into cortical neurons for disease modeling and regenerative medicine. However, these procedures are hard to provide sufficient cells for their applications. Using a combination of small-molecules and growth factors, we previously identified one condition which can rapidly induce hPSCs into neuroepithelial stem cells (NESCs). Here, we developed a scalable suspension culture system, which largely yields high-quality NESC-spheres and subsequent cortical neurons.
\end{abstract}

Methods: The NESC medium was first optimized, and the suspension culture system was then enlarged from plates to stirred bioreactors for large-scale production of NESC-spheres by a stirring speed of $60 \mathrm{rpm}$. During the expansion, the quality of NESC-spheres was evaluated. The differentiation potential of NESC-spheres into cortical neurons was demonstrated by removing bFGF and two pathway inhibitors from the NESC medium. Cellular immunofluorescence staining, global transcriptome, and single-cell RNA sequencing analysis were used to identify the characteristics, identities, purities, or homogeneities of NESC-spheres or their differentiated cells, respectively.

Results: The optimized culture system is more conducive to large-scale suspension production of NESCs. These largely expanded NESC-spheres maintain unlimited self-renewal ability and NESC state by retaining their uniform sizes, high cell vitalities, and robust expansion abilities. After long-term expansion, NESC-spheres preserve high purity, homogeneity, and normal diploid karyotype. These expanded NESC-spheres on a large scale have strong differentiation potential and effectively produce mature cortical neurons.

Conclusions: We developed a serum-free, defined, and low-cost culture system for large-scale expansion of NESCs in stirred suspension bioreactors. The stable and controllable 3D system supports long-term expansion of highquality and homogeneous NESC-spheres. These NESC-spheres can be used to efficiently give rise to cortical neurons for cell therapy, disease modeling, and drug screening in future.

Keywords: Human pluripotent stem cells, Neuroepithelial stem cells, Large-scale suspension culture, Cortical neurons

\footnotetext{
* Correspondence: litq@lpbr.cn

†Shumei Zhao and Kui Duan contributed equally to this work.

'Yunnan Key Laboratory of Primate Biomedical Research, Institute of Primate

Translational Medicine, Kunming University of Science and Technology, Kunming, China

${ }^{2}$ Xi'an ChaoYue Stem Cell Co, Ltd, Xi'an, China
}

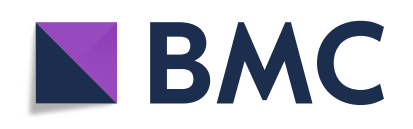

(- The Author(s). 2020 Open Access This article is licensed under a Creative Commons Attribution 4.0 International License, which permits use, sharing, adaptation, distribution and reproduction in any medium or format, as long as you give appropriate credit to the original author(s) and the source, provide a link to the Creative Commons licence, and indicate if changes were made. The images or other third party material in this article are included in the article's Creative Commons. licence, unless indicated otherwise in a credit line to the material. If material is not included in the article's Creative Commons licence and your intended use is not permitted by statutory regulation or exceeds the permitted use, you will need to obtain permission directly from the copyright holder. To view a copy of this licence, visit http://creativecommons.org/licenses/by/4.0/. The Creative Commons Public Domain Dedication waiver (http://creativecommons.org/publicdomain/zero/1.0/) applies to the data made available in this article, unless otherwise stated in a credit line to the data. 


\section{Background}

The cortex is a target for many disorders of the brain at all stages of life. For example, perturbation of cortical development can lead to neurodevelopmental disorders, such as autism spectrum disorders, while the adult cortex is a major site for certain neurodegenerative and injury diseases including Alzheimer's disease, frontotemporal dementia, stoke, and cortex injury. Currently, there is still lack of any effective treatment for these diseases. Due to the poor/limited plasticity of adult central nervous system (CNS), it is difficult to produce new neurons by activating endogenous neural stem cells (NSCs) to replace lost neurons [1-3]. Previous reports have shown that NSCs transplantation have a therapeutic effect on neurological diseases $[4,5]$ and even reconstruct the damaged neural network [6-10]. At present, several suspension culture systems of NPCs or NSCs have been established [11-14], but they cannot stably and reproducibly provide high-quality stem cells, such that they are difficult to meet the requirements of cell quality and quantity for cell transplantation. In addition, the differentiation potential of human pluripotent stem cells (hPSCs) in vitro also provides a unique opportunity to study normal and abnormal corticogenesis.

The cerebral cortex contains dozens of neuron subtypes and is the most complex structure in the mammalian brain. PSCs have been successfully induced into mature brain excitatory projection neurons by adding some small molecule compounds [15-17]. To simulate the development of the brain and the cortex, PSCs were formed into mini brain-like organoids by 3D suspension culture [18-20]. However, the limitations of these methods are poor controllability and difficulty in obtaining cortical cells in large quantities, which hinders their clinical applications in regenerative medicine. Although different systems have been established to directly induce iPSCs into neurons or cortical spheroids in the stirred suspension bioreactor, these neurons obtained by these methods lack of proliferation capabilities and cannot be passaged [21, 22]. Thus, the conventional adherent culture and direct cortical differentiation from hPSCs pose challenges to mass production of highquality cortical neurons and hamper the routine application of hPSC-derived lineages in the disease modeling and regenerative medicine.

During the development of mammalian brain, NSCs mainly go through two different developmental stages: a neuroepithelial stem cell (NESC) stage and a radial glial progenitor cell (RGPC) stage [23]. RGPCs are heterogeneous and have limited neurogenic differentiation [24, 25]. In contrast, NESCs are NSCs locating at the earlyformed neural tube stage with a strong self-renewal ability and differentiation potential and can extensively generate cortical neurons [26, 27]. These characteristics endow the NESCs as ideal donor cells for potential therapeutic applications in repairing lost cortical neurons. Using a combinatorial small molecule and growth factors, we previously identified one condition to rapidly differentiate hPSCs into NESCs [26, 28]. However, the large-scale suspension culture of NESCs has not been established, and the technology about the large-scale production of NESCs is still challenging. In addition, whether these NESCs could massively produce cortical neurons is unclear. In the study, we developed a scalable suspension-culture system to largely yield high-quality NESC-spheres. The suspension-culture system supported enlargement of NESC-spheres from plates or dishes to stirred bioreactors. Moreover, these NESCspheres efficiently produce mature cortical neurons.

\section{Materials and methods hESC culture}

Human embryonic stem cell lines hESC1 and BG02 were maintained on MEFs as previously described [29]. Among them, hESC1 is an embryonic stem cell line developed by our laboratory [30]. BG02 cell line was gifted from Pro. Zheng Lab of Kunming Institute of Zoology, Chinese Academy of Sciences. Human pluripotent stem cells H9 are suspension cultured in the AIC medium without feeders or matrigel [30]. The AIC medium [30] consisted of modified N2B27 medium supplemented with $10 \mathrm{ng} / \mathrm{ml}$ Activin A (Peprotech, 120-14E), $2 \mu \mathrm{M}$ IWP-2 (Selleck, S7085), and 0.6 $\mu$ M CHIR99021 (Selleck, S2924).

\section{Induce NESC production from hESCs}

hESCs were digested into small clumps for suspension culture on ultra-low attachment plates (Corning, 3471) in the NESC-derived medium [26], which is composed of Advance DMEM/F12 (Gibco, 10565-018): Neurobasal media (Gibco, 21103-049) (1:1) supplemented with $1 \%$ N2 (Gibco, 17502-048), 2\% B27 (Gibco, 17504-044), 1\% Glutmax (Gibco, 35050-061), 10 ng/mL OsrbFGF (Oryza sativa recombinant human basic fibroblast growth factor, Wuhan Healthgen, China, HYC005M01), $3 \mu \mathrm{M}$ CHIR99021 (Selleck, S2924), $5 \mu$ M SB431542 (Cellagen technology, C7243), $0.2 \mu \mathrm{M}$ Compound E (Calbiochem, 565790), $0.1 \mu \mathrm{M}$ LDN193189 (Selleck, S2618), and 0.1 mM $\beta$-mercaptoethanol (Sigma, M3148). After suspension culture for 6 days, neuron bodies (NBs) were digested into single cells and inoculated into ultra-low attachment plates with $\mathrm{CHbFSB}+\mathrm{LIF}$ culture medium. The CHbFSB+LIF culture medium $[26,28,31]$ is composed of Neurobasal medium, 1\% N2, 2\% B27, 1\% NEAA (Gibco, 11140-050), 1\% Glutmax, $3 \mu \mathrm{M}$ CHIR99021, $5 \mu \mathrm{M}$ SB431542, and $10 \mathrm{ng} / \mathrm{ml}$ OsrbFGF surplus with $1000 \mathrm{U} / \mathrm{ml}$ hLIF (Millipore, LIF1050). 
Suspension and long-term expansion of hNESC-spheres To extensively expand NESCs in vitro, NESCs were digested into single cells and cultured in ultra-low attachment plates. They were cultured in chemically defined $\mathrm{CHbFSB}+\mathrm{LIF}$ or CHbFSB culture medium. The CHbFSB culture medium consists of Neurobasal media surplus with $0.25 \% \mathrm{~N} 2,0.5 \% \mathrm{~B} 27,1 \% \mathrm{NEAA}, 1 \%$ Glut$\max , 3 \mu \mathrm{M}$ CHIR99021, $5 \mu \mathrm{M}$ SB431542, and $10 \mathrm{ng} / \mathrm{ml}$ OsrbFGF. TrypLE ${ }^{\mathrm{Tn}}$ Express Enzyme (Gibco, 12,605,028) was diluted for 2 times with PBS (Sigma, D5652) to digest NESCs for encouraging cell propagation when passaging. NESCs were routinely passaged at $1: 3$ to $1: 4$ ratios every 3 days.

\section{Large-scale expansion of hNESC-spheres}

Digested hNESCs (passage 19) were inoculated into a $125 \mathrm{ml}$ suspension bioreactor (Wiggens, BIOMIX Control MS4) with a 100-ml CHbFSB medium at the cell density of $3 \times 10^{5}$ cells $/ \mathrm{ml}$. Every 3 days, the NESCspheres were dissociated and passaged using TrypLE ${ }^{\text {тx }}$ Express Enzyme: PBS (1:2). The agitation rate of NESCs growing in a stirred suspension bioreactor is $60 \mathrm{rpm}$. The bioreactor was housed in a humidified incubator with $5 \% \mathrm{CO} 2$ at $37^{\circ} \mathrm{C}$. The NESCs were fed 2 days after inoculation by replacing $50 \%$ of the medium with the fresh medium.

\section{Transcriptome analysis}

Total RNA was isolated from NESC-spheres cultured in the CHbFSB+LIF or CHbFSB medium using the RNeasy Mini Kit (QIAGEN, 74106). RNA sequencing libraries were constructed using the NEBNext ${ }^{\circ}$ Ultra RNA Library Prep Kit for Illumina ${ }^{\circ}$ (NEB England BioLabs, E7530L). The fragmented and randomly primed $2 \times 150$ bp paired end libraries were sequenced using an Illumina HiSeq $\mathrm{X}$ Ten. The generated sequencing reads were mapped against human genome build hg38 using HISAT2 alignment software tools. The read counts for each gene had calculated and normalized with StringTie software [32]. For subsequent analysis of gene expression, genes were retained in both datasets if they were expressed in at least one sample with an FPKM > 5 threshold. Heat maps were generated using pheatmap package in the R software (https://www.r-project.org/).

\section{0x single-cell gene expression analysis}

We performed RNA amplifcation of single cell from hNESC-spheres with the 10X Genomics plaform. Nine thousand seven hundred sixty-nine single cells were sequenced with the Illumina NextSeq 500. The raw data were first analyzed by Cellranger. The output of the Cellranger (v3.1) was used to create a Seurat object with Seurat packages (v3.0) [33]. The UMAP non-linear dimensional reduction techniques were used to visualize and cluster analysis based on the same PCs input. The VlnPlot tool was used to show marker gene expression probability distributions across clusters. The FeaturePlot tool was used to visualize feature expression on UMAP plots. The differentially expressed genes between two specific groups of cells have been performed with FindMarkers function based on the non-parameteric Wilcoxon rank sum test.

\section{Cortical neuron differentiation of hNESC-spheres}

To induce differentiation, hNESC-spheres were digested into single cells and cultured on plates coated with laminin $(5 \mu \mathrm{g} / \mathrm{ml})$ and poly-ornithine (Sigma, $15 \mu \mathrm{l} /$ well) in the differentiation medium. The differentiation medium [26] was composed of Neurobasal, $0.5 \%$ N2, 1\% B27, $1 \%$ NEAA, and $1 \%$ Glutmax. The medium was replaced by the fresh medium every 3 days. On day 6 postdifferentiation (pdD6), $10 \mathrm{ng} / \mathrm{ml}$ BDNF (Gibco, PHC7074) and $10 \mathrm{ng} / \mathrm{ml}$ GDNF (Gibco, PHC7044) were added into the medium to induce terminal maturation of neurons. To get more mature neurons, $2 \times 10^{3} / \mathrm{cm}^{2}$ mouse astrocytes were co-cultured with the differentiated neurons at pdD6. Mouse astrocytes were isolated from new-born mouse cortex. Before used for co-culture with neurons, astrocytes were cultured for four passages to exclude the contamination of neurons.

\section{Immunocytochemistry}

Cells were fixed with $4 \%$ paraformaldehyde for $20 \mathrm{~min}$, washed with PBS for three times, treated with $0.2 \%$ Triton X-100 (sigma, X100) for $30 \mathrm{~min}$, washed with PBS for three times, incubated in blocking buffer (3\% BSA (Generay, 9048-46-8) in PBS) for $30 \mathrm{~min}$ at room temperature, and washed with PBS for three times. The cells were then incubated with primary antibody overnight at $4{ }^{\circ} \mathrm{C}$ (Table S1). The cells were washed with PBS including $0.05 \%$ Tween 20 for three times and incubated with Alexa 488, 568, or 647 Fluor-conjugated secondary antibodies (Thermo Fisher: goat anti-rabbit, A31573; goat anti-mouse, A10037; donkey-anti-goat, A11055; Jackson ImmunoResearch: donkey anti-chicken, 703$545-105 ; 1: 600)$ for $2 \mathrm{~h}$ at room temperature. Nuclei were visualized by DAPI staining (Sigma, 32,670).

\section{Cryosections of hNESC-spheres}

hNESC-spheres from different generations (passages 5, $15,25)$ were harvested at the day 3 of culture. hNESCspheres were fixed with $4 \%$ paraformaldehyde for $30 \mathrm{~min}$ and washed with PBS for three times. They were dehydrated by adding $20 \%$ sucrose for $10 \mathrm{~min}$, embedded in O.C.T (Optimum Cutting Temperature Compound, Sakura, 4583), and frozen at $-20^{\circ} \mathrm{C}$. These samples were cut into $10-\mu \mathrm{m}$-thick slices and then subjected to immunofluorescence staining. 


\section{Statistical analysis}

All of experiments including immunocytochemistry were at least performed repeated 3 times. Image J and GraphPad prism software were used to measure the diameters of NESC-spheres over culture. Quantification data were represented as mean \pm standard deviation (SD). Comparison of the outcome of variables in various experiments was assessed by unpaired Student's $t$ test. $P$ value of $<0.05$ was considered as significance.

\section{Results}

Establishing a suspension culture system of human NESCs Following our previous protocol (Fig. 1a) [26, 28], we successfully induced hESCs into highly enriched NESCs using a cocktail including bFGF, CHIR99021 (a GSK3 inhibitor), SB431542 (a transforming growth factor $\beta$ inhibitor), Compound E (a Notch inhibitor), and LDN193189 (an inhibitor of ALK2 and ALK3) [34-37]. Using this system, hPSCs in suspension culture in the AIC medium [30] were also successfully induced into NESCs (Fig. S3A-C). Considering that suspension-culture is a key to achieve a large-scale production of cells, we sought to develop a suitable suspension-culture system of NESCs. Our previous works showed that the CHbFSB+ LIF medium (including bFGF, LIF, CHIR99021, and SB431542 supplemented with 1\% N2 and 2\% B27) allows clonal expansion of hESC-derived NESCs to develop into miniature neural tube (NT)-like structures in the adherent culture $[26,28]$. In our initial suspension-culture, NESCs at post-differentiation day 6 (pdD6) were digested into single cells and subjected to $3 \mathrm{D}$ suspension propagation in the CHbFSB+LIF medium. As expected, single hNESCs grown in the CHbFSB+LIF quickly formed NESC-spheres at day 1 and exhibited strong proliferation in the next 2 days (Fig. 1b).

Given that N2 and B27 supplements are costly and usually used to culture differentiated neurons, we guessed that the low concentration of N2 and B27 may be beneficial to NESCs expansion. Based on the recommended concentration of the manufacturer, we diluted the N2 and B27 four times, respectively. An our previous study together with another report have showed that the removal of leukemia inhibitory factor (LIF) from the medium cannot affect the human NESCs or NSCs survival and self-renewal [28, 38], suggesting that selfrenewal of NESC-spheres at high cell density may not require exogenous LIF. Thus, we designed the $\mathrm{CHbFSB}$ medium (including bFGF, CHIR99021, and SB431542 as well as $0.25 \% \mathrm{~N} 2$ and $0.5 \%$ B27 supplements) as an optimized medium. Next, we evaluated the expansion of NESC-spheres in the CHbFSB medium. Interestingly, the CHbFSB medium could efficiently support suspension propagation of NESC-spheres (Fig. 1b-f). At day 3, these NESC-spheres were subjected for passaging every
3 days and routinely passaged at 1:4 to 1:6 with a cell density of $3.0 \times 10^{5}$ per milliliter. The NESC-spheres displayed round or ellipsoid morphologies and maintained uniform size and high viability over passaging (Fig. 1c-e).

Next, we compared the growth of NESC-spheres in the two systems. Quantification showed that the diameters of NESC-spheres at day 3 were approximately $124.05 \pm 20.00 \mu \mathrm{m}$ for the CHbFSB+LIF and $146.10 \pm$ $28.34 \mu \mathrm{m}$ for the CHbFSB, respectively (Fig. 1c). Longterm evaluation showed that uniform sizes of NESCspheres were stably maintained in the two culture systems over passaging (Fig. 1d). Above 95\% of cell viability in NESC-spheres was retained in the two culture systems (Fig. 1e). Cell yield was up to $3 \sim 5$-fold $(\mathrm{CHbFSB}+$ LIF) or 4 6-fold (CHbFSB) every passage (3 days). These cells showed exponential growth over serial passages, resulting in the increase to $1.07 \times 10^{11}$ cells $(\mathrm{CHbFSB}+$ LIF) versus $1.39 \times 10^{12}$ cells $(\mathrm{CHbFSB})$ within 30 days without losing obvious proliferative capacity, respectively (Fig. 1f). These results showed that the optimized medium better promoted the proliferation of NESCs, but had no significant $(p>0.05)$ effect on the cell viability of NESCs.

\section{Human NESC-spheres maintained NESC identity during long-term expansion}

NESCs generally exist in the embryonic neural plate and neural tube and have strong ability to divide and proliferate $[16,20]$. SOX1 is one of the molecular proteins capable of labeling early NESCs [39, 40]. In addition, NESCs specifically express neural precursor markers (PAX6, SOX2, and NESTIN) and a polar molecular marker tight junction protein (ZO-1). Immunofluorescence for NESC-spheres from different passages showed that NESCs growing in the two systems uniformly expressed PAX6, SOX1, NESTIN, and SOX2, but not glial cell marker GFAP (Figs. 2a, S1A, S2A and S3D). It is noted that the NESC-spheres culturing in the CHbFSB system had fewer differentiated $\mathrm{TUJ}^{+}$neurons than those in the CHbFSB+LIF system (Fig. 2a, e). However, no significant difference in the expression of SOX1, SOX2, and PAX6 was observed in the NESC-spheres from the two culture systems (Fig. $2 b-d$ and S2B). Together, the CHbFSB system better supported the longterm expansion of NESC-spheres than the CHbFSB+LIF. Therefore, we used the CHbFSB for subsequent experiments unless otherwise noted.

We next explored whether NESCs from spheres maintain the ability to self-organize into NT-like structures by clone formation assays of single cells (Fig. 2f). NESCspheres were digested into single cells and seeded into 96-well plates (Fig. 2g). NESCs self-organized into NTlike structures at day 12 (Fig. 2h). These NT-like structures uniformly expressed NESTIN, SOX2, SOX1 and 


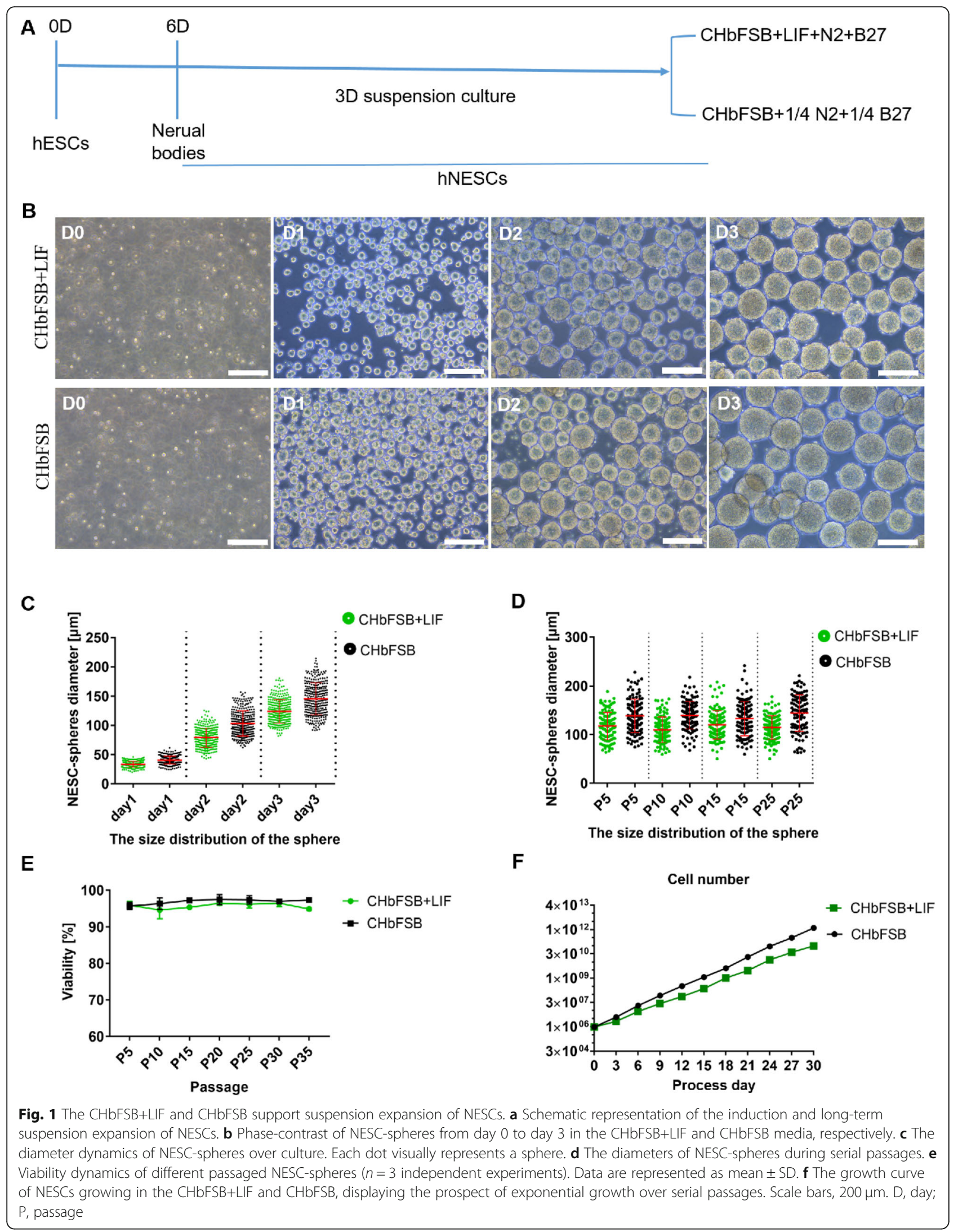




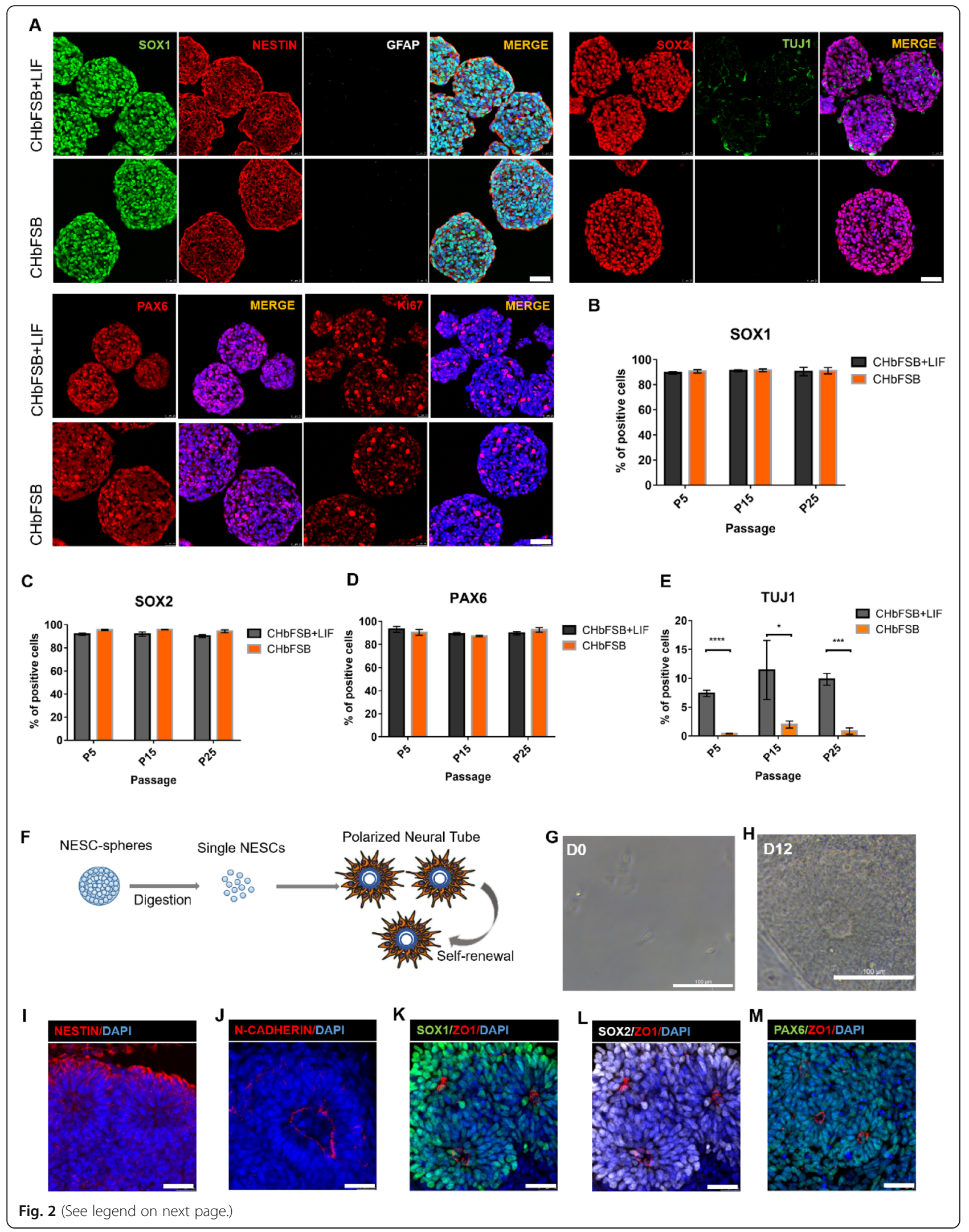


(See figure on previous page.)

Fig. 2 Long-term expanded NESC-spheres maintained NESC characteristics. a Immunofluorescence staining of cryosections showed that NESCspheres from passage 5 at day 3 express NESC markers, such as SOX1, NESTIN, SOX2, and PAX6, and the proliferation-associated marker Ki-67. In contrast, NESC-spheres are negative for GFAP and express TUJ1 in a small number of cells. b-d Quantification of the number of SOX1 (b)-, SOX2 (c)-, and PAX6 (d)-positive cells in the passage 5, 15, 25 NESC-spheres, respectively. e Quantification of differentiated TUJ1-positive neurons in the passaged 5, 15, 25 NESC-spheres, respectively. $\mathbf{f}$ Schematic representation of single NESCs from spheres self-organization into neural tube (NT)-like structures in the colony assays. $\mathbf{g}$ Single NESCs in one well of the 96-well plate at day 0 . $\mathbf{h}$ Representative NT-like structures of NESCs at day 12 . i$\mathbf{m}$ The polarized NTs express NESC markers, such as NESTIN, N-CADHERIN, SOX1, SOX2, PAX6, and ZO-1. b-e Data are represented as mean \pm SD ( $n=3$ independent experiments). ${ }^{*} p<0.05,{ }^{* * *} p<0.001,{ }^{* * *} p<0.0001$ by Student's $t$ test. Scale bars: $\mathbf{g}, \mathbf{h} 100 \mu \mathrm{m} ;$ others, $50 \mu \mathrm{m}$

PAX6, clustered ZO-1 and N-CADHERIN marking the luminal side (Fig. 2i-m). Together, NESCs after extensive expansion still retained the NESC identity.

\section{Large-scale expansion of human NESC-spheres}

To test the potential of large-scale culture, NESCspheres expanded in ultra-low attachment plates were digested into single cell suspensions and inoculated in a $125 \mathrm{ml}$ suspension bioreactor with the stirring rate of 60 rpm (Fig. 3a). As expected, NESCs proliferated normally and formed aggregates in the bioreactor (Fig. 3b). On day 3, average diameters of these NESC-spheres increased up to approximately $156.97 \pm 32.78 \mu \mathrm{m}$ (Fig. 3c) and NESCs held $>95 \%$ of cell viability when digested into single cells for passaging (Fig. 3d). This stirring suspension culture facilitated serial passages of NESCspheres because of easily controlling sphere diameter and minimizing cell apoptosis in the sphere center. In the bioreactor, NESC-spheres maintained extensive proliferation and NESC identity for at least 23 passages and remained a normal diploid karyotype (Fig. 3e). Immunostaining showed that NESC-spheres expressed SOX1, NESTIN, SOX2, and PAX6 (Fig. 3f, g). No expression of GFAP and TUJ1 indicated the absence of glial cell or neuron differentiation in NESC-spheres (Fig. 3f). The wide expression of Ki-67 further revealed the strong proliferation of NESCs (Fig. 3f). The above data showed that the system was suitable for large-scale production of NESCs in stirred suspension bioreactors.

\section{Molecular and cellular homogeneity of human NESC- spheres}

To identify NESC-spheres features, the global transcriptomes of hNESC-spheres growing in different culture systems including two-dimensional adherent (2D) and three-dimensional suspension (3D-CHbFSB+LIF and 3D-CHbFSB) were analyzed via RNA sequencing (RNAseq). Sample correlation (Spearman) showed that the transcriptomes of NESCs between cell lines clustered to closer than that different culture conditions (Fig. 4a). We found that NESC-spheres highly expressed many genes, such as LIN28A [41], ASNS [42], LMNB1 [43], ZIC2 [44], AXIN2 [45], LYAR [46], and LEF1, which have been reported to play critical roles in NT development (Fig. 4b). In contrast, genes relative to RGC, IPC, and neuron displayed no or low expression under all culture conditions (Fig. 4b). Together, the transcriptome data confirmed the NESC identity of NESCspheres over extensive passaging.

To further monitor cell homogeneity after long-term expansion of NESC-spheres, we performed highthroughput single-cell RNA-sequencing (scRNA-seq) for late-passage cell populations using a 10x-genomics platform. We digested the NESC-spheres from passage 28 into single cells, detected 9769 cells with 4425 genes and analyzed their molecular characteristics. The analysis identified 3 distinct cell clusters, a NESC cluster accounting for $94.58 \%$ of the total cell population, a DPC (differentiating-prone cell) cluster (1.81\%), and an IPC (intermediate progenitor cell) cluster (3.61\%), based on their gene expression patterns (Fig. 4c). Cell-typeenriched genes were identified by comparing each cluster. NESCs uniquely expressed NESC genes, such as LIN28A, ASNS, LMNB1, PAX6, SOX2, and NES/NESTIN (Fig. 4d). DPCs are found to express $S O X 2$ but not PAX6 (Fig. 4e), indicating these cells in the cluster were locating in the differentiating routine. In contrast, IPCs expressed the IPC marker genes NHLH1 and PPP1R17 and partially expressed $D C X$ (a neuron marker) (Fig. 4f, g). In addition, all cells did not express the astrocyte marker GFAP and the cerebral cortex neuron markers SATB2 and TBR1 (Fig. 4g). These results demonstrate that cells in largely expanded NESC-spheres are highly homogenous.

\section{Large-scale expansion of NESC-spheres generated mature cortical neurons}

In the early stages of brain development, NESCs undergo self-renewal through symmetric division or differentiate into neurons by asymmetric division [47]. The cerebral cortex is composed of six layers in anatomical physiology, and layer-specific neurons are generated by sequence. The first layer, II/III/IV layer (intracortical projection neurons), and V/VI layer (corticofugal projection neurons) are defined into the Cajal-Retzius, upper, and deeper layers, respectively $[16,48,49]$. To generate cortical neurons, NESCs were induced to differentiation by removing bFGF, CHIR99021, and SB431542. At day 6 


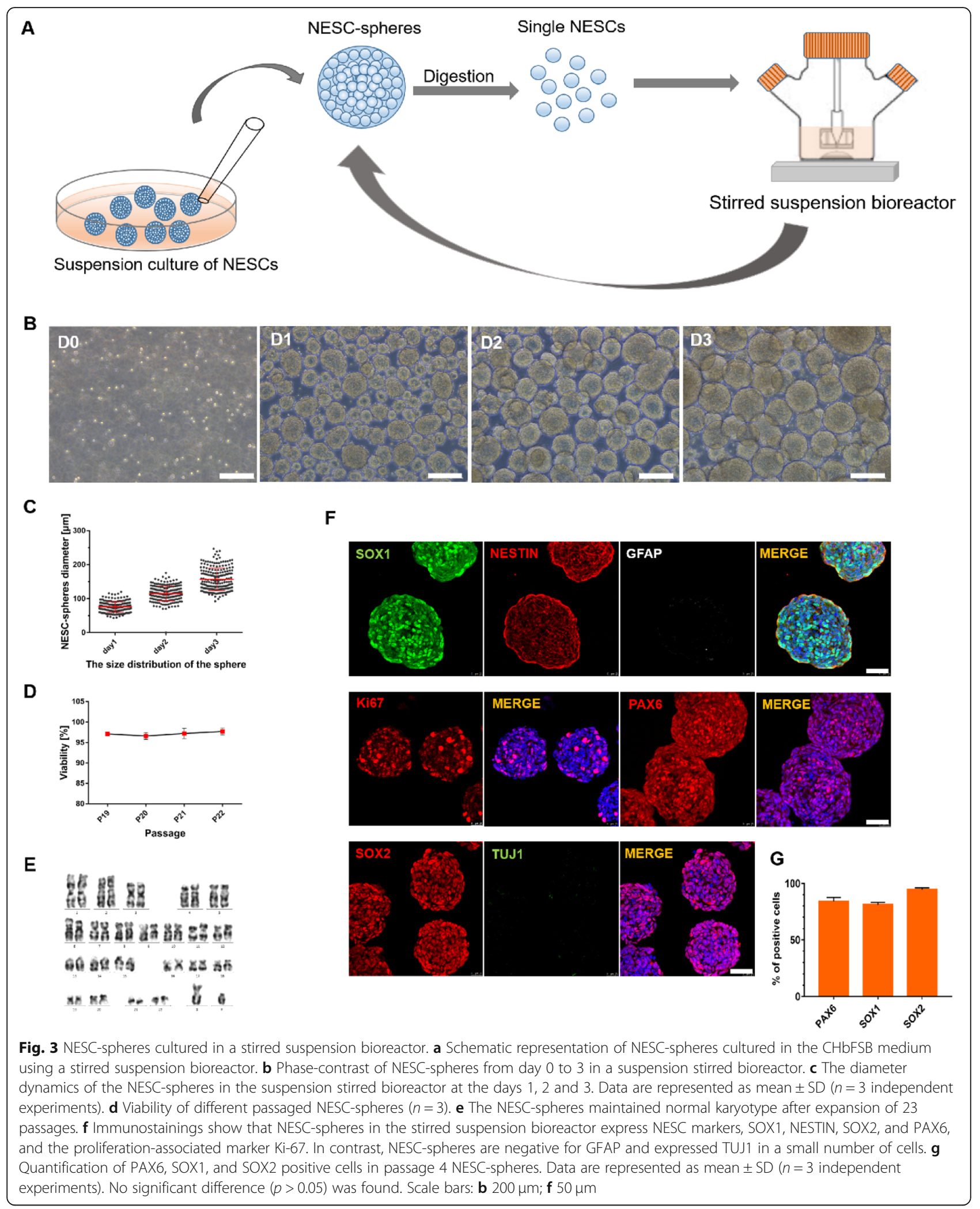

post-differentiation (pdD6), the mouse astrocytes were added into the dishes and co-cultured with differentiated neurons. At pdD 34, more than $90 \%$ of differentiated cells from NESCs co-expressed TUJ1 and human nuclei (HN) (Fig. 5a). Furthermore, long-term suspensionexpansion did not destroy the differentiation potentials 

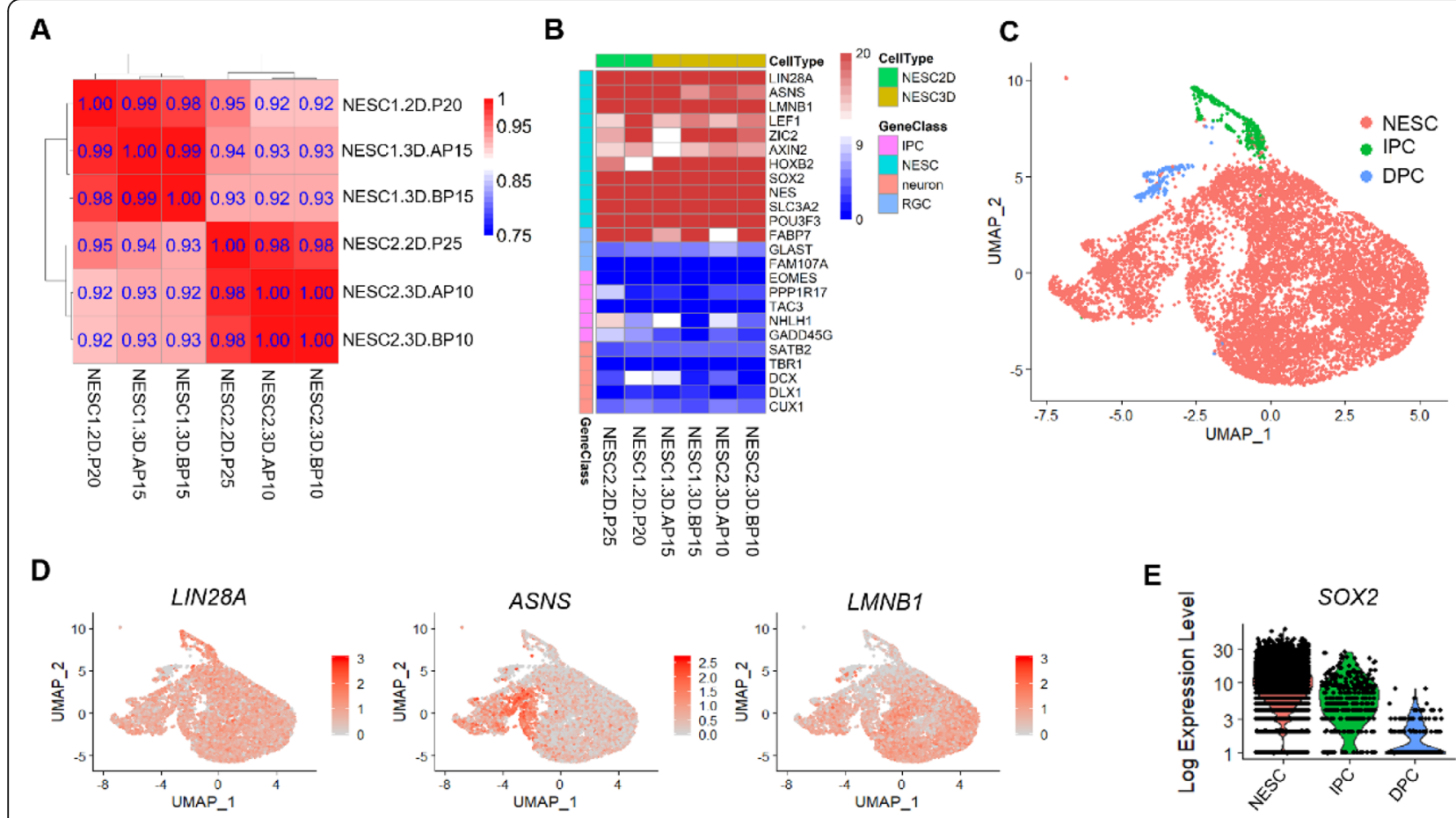

E
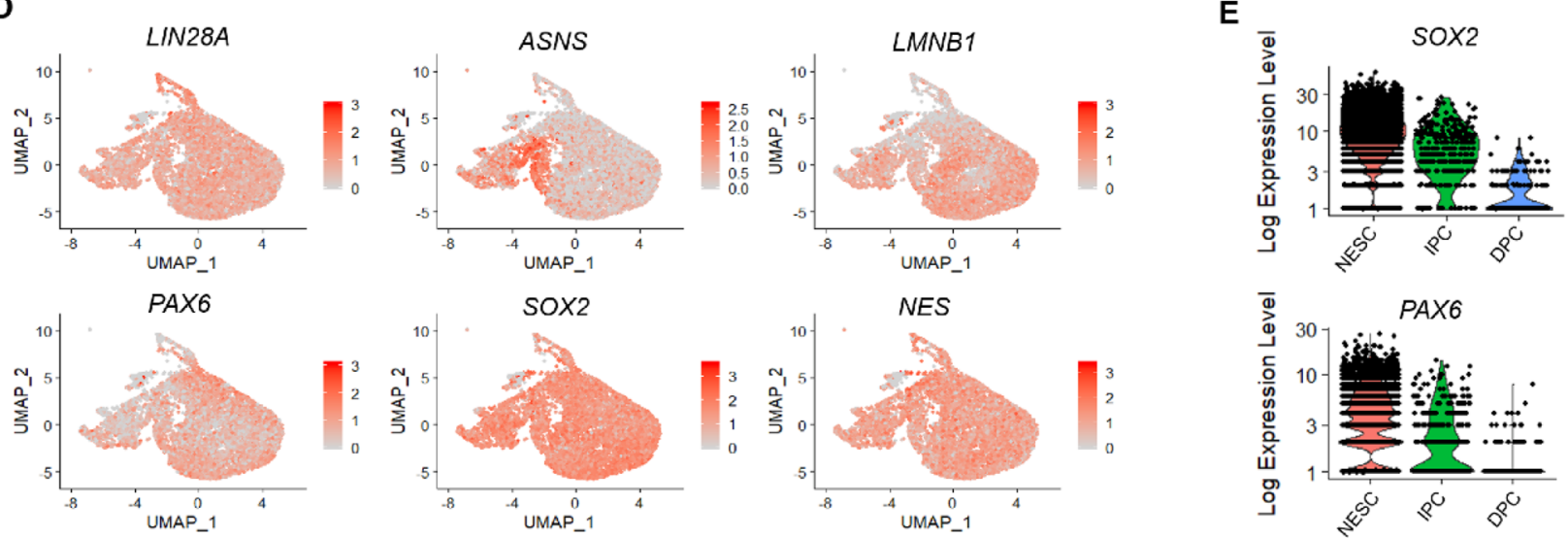

F

$\mathbf{G}$
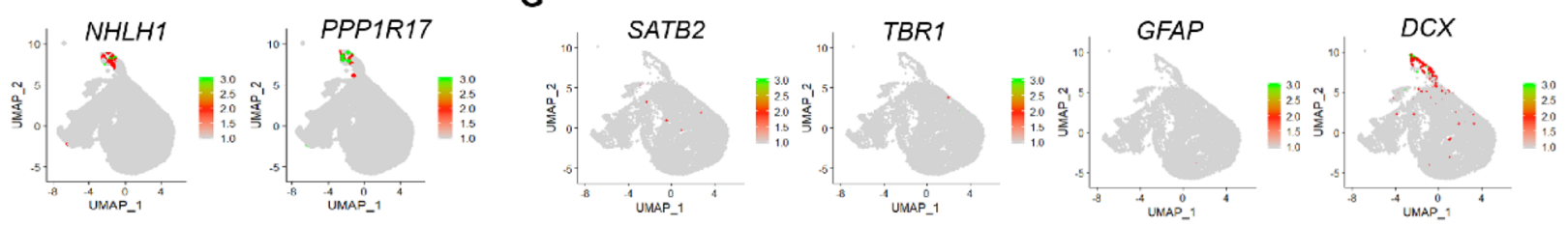

Fig. 4 Molecular and cellular homogeneity of NESC-spheres. a Sample correlation (Spearman) of transcriptomes among the NESC1-2D-P20, NESC2-2D-P25, NESC1-3D-AP15, NESC2-3D-AP10, NESC1-3D-BP15, and NESC2-3D-BP10. Two cell lines were analyzed and termed as NESC1 (from hESC1) and NESC2 (from BG02). 2D, two-dimensional culture; 3D, three-dimensional suspension-culture; P, passage. A, the CHbFSB+LIF medium; B, the CHbFSB medium. $\mathbf{b}$ The heatmap of genes relative to NESCs, radial glial cells (RGCs), intermediate progenitor cells (IPCs), and neurons. $\mathbf{c}-\mathbf{g}$ A comprehensive transcriptome analysis of single cells using 10x genomics platform. c Uniform manifold approximation and projection (UMAP) plots of 9769 cells from NESC-spheres (P28) colored by cluster annotation. Dots, individual cells. Red, NESCs; green, IPCs (intermediate progenitor cells); blue, DPCs (differentiating-prone cells). $\mathbf{d}$ NESCs are colored according to the expression levels of the indicated markers on the UMAP map (red, high; gray, low). e Violin plots show expression levels and distributions of SOX2 and PAX6 in different three clusters. f, g UMAP plots of cells colored by expression of selected marker genes relative to astrocytes, neurons, and IPCs. The different colors indicate the difference of expression level (green, high; gray, low)

of NESCs (Fig. 5b). After co-cultured with mouse astrocytes, NESCs differentiated into cortical neurons including the BRN2- and CUX1-positive upper layer neurons (Fig. 5c, f), as well as FOXP2- and CTIP2-positive deeper layer neurons [17, 50] (Fig. 5d, e). Quantification of differentiated cells at pdD42 showed that FOXP2-positive neurons from passage 8 (P8) and passage 30 (P30) NESC-spheres were $48.29 \pm 5.03 \%$ and $39.17 \pm 9.89 \%$, respectively (Fig. $5 \mathrm{~d}, \mathrm{~g}$ ), while BRN2-positive neurons at pdD42 accounted for $34.82 \pm 3.88 \%$ and $40.14 \pm 4.09 \%$, respectively (Fig. 5c, g). These differentiated neurons expressed TBR1, a marker of cortical projection neurons (Fig. S4A). In addition, we also detected a few REELIN ${ }^{+}$ (a marker of Cajal-Retzius neurons) neurons and SATB2 $^{+}$(a marker of layer V- or upper-layer callosal neurons) neurons (Figs. 5h and S4A). 


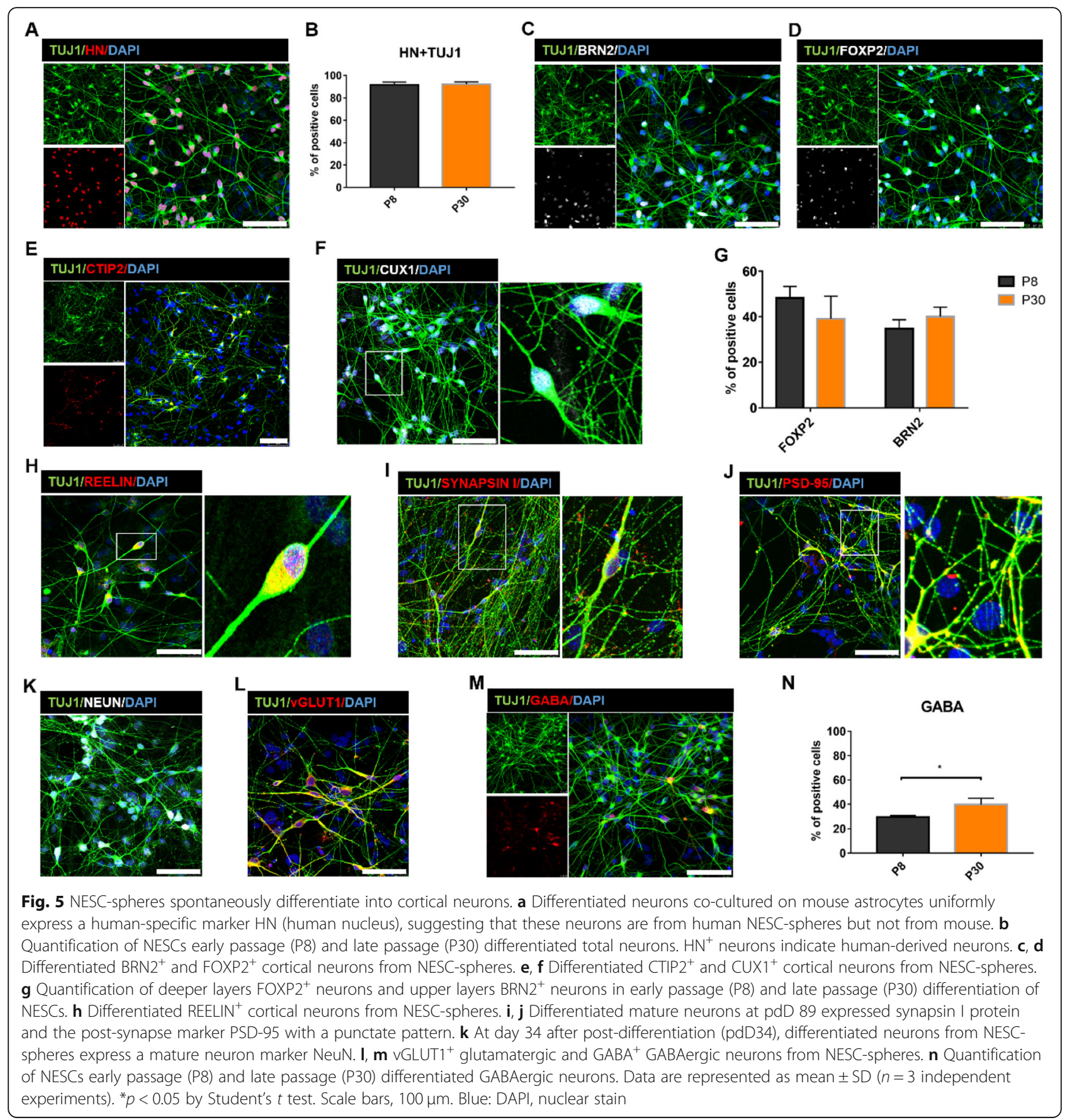

Synapse genesis is a key step in the formation of neural circuits. To test whether these cortical neurons can form physical synapses in vitro, differentiated neurons were cocultured with mouse astrocytes. Most of differentiated neurons expressed NeuN at pdD34 (Fig. 5k), pre-synaptic protein SYNAPSIN I and post-synaptic protein PSD-95 in their axons in a punctate pattern at pdD89 (Fig. 5i, j). In the cerebral cortex, the vast number of neurons is mainly made up of two types according to secreted neurotransmitters: more than $80 \%$ of glutamic-excitatory (glutamatergic) projection neurons and about $20 \%$ of GABA aminobutyric acid-inhibitory (GABAergic) neurons [51]. Subtype identification showed that NESCs spontaneously differentiated into glutamatergic neurons and GABAergic neurons (Fig. 5l, m). Quantification data showed that NESCs at passage 8 and 30 gave rise to $29.74 \pm 1.26 \%$ and $39.78 \pm 5.17 \%$ of GABAergic neurons, respectively (Fig. 5n). Moreover, these of GABAergic neurons co-expressed calretinin (CR), calbindin (CB), or parvalbumin (PV) (Fig. S4B-D), confirming the identity of inhibitory interneurons. 


\section{Discussion}

Here, we developed a system for large-scale production of NESCs derived from hESCs in a stirred suspension bioreactor. Our NESCs stably maintain long-term and large-scale expansion and efficiently give rise to cortical neurons. These NESC-spheres have unique characteristics: (1) retain above $95 \%$ of cell viability and uniform size; (2) have robust expansion ability; (3) express NESC markers, such as PAX6, SOX1, NESTIN, and SOX2; (4) express many NT genes, such as LIN28A, ASNS, and $L M N B 1$, but not glial cell marker GFAP; (5) selforganize into miniature NT-like structures and cluster tight junction protein ZO-1 and N-CADHERIN into apical side; (6) display high homogeneity of NESCs in spheres; and (7) give rise to cortical neurons.

Neurosphere culture systems are useful for biological studies of developmental processes [52, 53]. Stirred bioreactor suspension culture is an effective way to expand stem cells for regenerative medicine and drug development $[54,55]$. However, the content of NSCs in cultured neurospheres is variable and depends on the stage of culture. Previous studies have reported high content of NSCs shortly after separation, but stem cell purity gradually declines in subsequently expanded subcultures [56, 57]. In the study, we developed the medium for the long-term expansion of NESC-spheres. Single-cell transcriptome data demonstrated that the percentage of NESCs in the expanded spheres after long-term passage was as high as 95\%. This system provides controlled, stable, and high-quality donor cells for further differentiation and treatment of neurological diseases.

Over the past few decades, several NPCs or NSCs culture systems have been established [11, 12, 14, 35, 5860], but these developed methods are difficult to provide a sufficient number of homogeneous NSCs for further differentiation, disease study, or clinical stem cell therapy. We previously established a feasible adherent culture system, including LIF, $1 \% \mathrm{~N} 2$, and $2 \% \mathrm{~B} 27$, of hNESCs [26, 28]. However, LIF is not required for the long-term expansion of NSCs or NESCs [28, 38]. According to manufacturer's suggestion, N2 supplement is recommended for growth and maturation of postmitotic neurons and B27 supplement increases neuronal survival, implying that their recommended concentrations may result in the neuron differentiation. As expected, we observed that $1 \% \mathrm{~N} 2$ and $2 \%$ B27 increased the $\mathrm{TUJ} 1^{+}$neurons production, whereas four-time dilutions decreased the $\mathrm{TUJ} 1^{+}$neurons differentiation. The further optimization N27 and B27 concentration may improve the suspension-culture system. Compared with other previous suspension culture methods [12, 13], our large-scale suspension system has three advantages. First, the system is defined and lower costs and enables NESC-spheres to stably maintain self-renewal during the long-term expansion. Second, NESC-spheres display uniform size and cell homogeneity along with the typical NESC identities. Third, NESC-spheres can effectively differentiate into cortical neurons. Based on these unique features, it strongly supports the ability to massively generate cortical neurons for drug screening and disease treatment.

During development, the human cortex is composed of six-layer projection neurons [16, 48, 49]. Adult cerebral cortex is poorly plastic, and it is difficult to produce nerve regeneration once damaged. Many neurological diseases, including epilepsy, autism, schizophrenia, and possibly Alzheimer's disease, are thought to result, at least in part, from the dysfunction of cortical interneuron [8]. Our scaled-up NESC-spheres have strong differentiation ability. NESC-spheres spontaneously initiated differentiation by removing growth factors and inhibitory molecules, and more than $90 \%$ of differentiated cells were neurons, including corticofugal projection neurons (deep-layer) and intracortical projection neurons (upper-layer). Interestingly, we also found that NESC-spheres can differentiate into cortical GABAergic interneurons. Calretinin and parvalbumin subtype neurons are present in both the middle ganglia and caudal ganglionic eminence at mouse E13.5, while calbindin neurons originate from the caudal ganglionic eminence [61-63]. Our experiments also showed that these three subtypes of interneurons could be specified from NESCspheres, providing an ideal platform to study interneuron development. One of main goals for the modern neuroscience is to restore neurological and cognitive functions after brain damage. NESCs have been regarded as a possible donor cells for nerve grafts to repair damaged neural circuits [64]. We confirmed that adherent cultured NESCs are capable of differentiating into functional neurons and integrating into the host cerebral cortex after grafted into mouse and monkey brains [26, 31]. Long-term continuous observation of the host did not show tumors after transplantation, implying that NESCs as donor cells have obvious effects and safety on stem cell therapy $[26,31]$. Thus, the efficient and economically viable hNESC-sphere scale-up system developed by us provides a stable and abundant source of cortical cells for cell therapy. However, subsequent works will be performed to confirm whether NESCsphere differentiation in vivo can replace endogenous injured nerve cells and reconstruct neural networks.

\section{Conclusions}

In summary, we developed a serum-free, defined, and low-cost culture system for large-scale expansion of NESCs with high quality, purity, and homogeneity in stirred suspension bioreactors. These NESC-spheres stably maintain long-term and large-scale through 
maintenance of unlimited self-renewal and NESCs state. Importantly, NESC-spheres effectively produce different subtypes of mature cortical neurons. This 3D-system overcomes the barriers of mass production of NESCs and can be used in commercial-level cortical neurons production for cell therapy, disease modeling, and drug screening in future.

\section{Supplementary information}

Supplementary information accompanies this paper at https://doi.org/10. 1186/s13287-020-01939-6.

\section{Additional file 1}

\section{Abbreviations}

NESCs: Neuroepithelial stem cells; NSCs: Neural stem cells; NPCs: Neural precursor cells; hPSCs: Human pluripotent stem cells; bFGF: Basic fibroblast growth factor; RNA-seq: RNA sequencing; CNS: Central nervous system; 2D: Two-dimensional; 3D: Three-dimensional; ESCs: Embryonic stem cells; RGPCs: Radial glial progenitor cells; NT: Neural tube; pdD: Post-differentiation day; LIF: Leukemia inhibitory factor; scRNA-seq: Single-cell RNA-sequencing; UMAP: Uniform manifold approximation and projection;

DPCs: Differentiation-prone cells; IPCs: Intermediate progenitor cells

\section{Acknowledgements}

We are grateful to members of the Li laboratory for their technical expertise and the project discussions.

\section{Authors' contributions}

T.L. conceived the idea for this project and designed and conducted the experiments. S.Z. and T.L. wrote the manuscript with help from all of the other authors. S.Z., K.D., Z.A., B.N., Y.C., and R.K. performed the experiments and analyzed data under the supervision of T.L.; K.D. analyzed the RNA sequencing data. All authors read and accepted the manuscript. The authors read and approved the final manuscript.

\section{Funding}

This work was supported by the Yunnan Key R\&D Program (2018ZF007-02 and 2018FA008), the National Key Research and Development Program of China (2018YFA0108500), the National Natural Science Foundation of China (31760268 and 81660266), the Yunnan Provincial Innovation Team (2018HC003), and Yunnan Fundamental Research Projects (2019FY002).

\section{Availability of data and materials}

All data generated or analyzed during this study are included in this published article and in supplementary figures.

\section{Ethics approval and consent to participate} Not applicable.

\section{Consent for publication}

Not applicable.

\section{Competing interests}

The authors declare that they have no competing interests.

Received: 24 April 2020 Revised: 22 July 2020 Accepted: 17 September 2020 Published online: 02 October 2020

\section{References}

1. Gates MA, Fricker-Gates RA, Macklis JD. Reconstruction of cortical circuitry. Prog Brain Res. 2000;127(127):115-56.

2. Nakamura $\mathrm{M}, \mathrm{Okano} \mathrm{H}$. Cell transplantation therapies for spinal cord injury focusing on induced pluripotent stem cells. Cell Res. 2013;23(1):70-80.

3. Fujimoto $Y$, Abematsu M, Falk A, Tsujimura $K$, Sanosaka $T$, Juliandi $B$, et al. Treatment of a mouse model of spinal cord injury by transplantation of human induced pluripotent stem cell-derived long-term self-renewing neuroepithelial-like stem cells. Stem Cells. 2012;30(6):1163-73.

4. Gupta N, Henry RG, Strober J, Kang SM, Lim DA, Bucci M, et al. Neural stem cell engraftment and myelination in the human brain. Sci Transl Med. 2012; 4(155):155ra37.

5. Uchida N, Buck DW, He D, Reitsma MJ, Masek M, Phan TV, et al. Direct isolation of human central nervous system stem cells. Proc Natl Acad Sci U S A. 2000;97(26):14720-5.

6. Daadi MM, Grueter BA, Malenka RC, Redmond DE Jr, Steinberg GK. Dopaminergic neurons from midbrain-specified human embryonic stem cell-derived neural stem cells engrafted in a monkey model of Parkinson's disease. PLoS One. 2012;7(7):e41120.

7. Gaillard A, Prestoz L, Dumartin B, Cantereau A, Morel F, Roger M, et al. Reestablishment of damaged adult motor pathways by grafted embryonic cortical neurons. Nat Neurosci. 2007;10(10):1294.

8. Southwell DG, Nicholas CR, Basbaum Al, Stryker MP, Kriegstein AR, Rubenstein $J$, et al. Interneurons from embryonic development to cellbased therapy. Science. 2014;344(6180):1240622.

9. Michelsen KA, Acosta-Verdugo S, Benoit-Marand M, Espuny-Camacho I, Gaspard N, Saha B, et al. Area-specific reestablishment of damaged circuits in the adult cerebral cortex by cortical neurons derived from mouse embryonic stem cells. Neuron. 2015;85(5):982-97.

10. Ma L, Hu B, Liu Y, Vermilyea SC, Liu H, Gao L, et al. Human embryonic stem cell-derived GABA neurons correct locomotion deficits in quinolinic acidlesioned mice. Cell Stem Cell. 2012;10(4):455-64.

11. Sun T, Wang X-J, Xie S-S, Zhang D-L, Wang X-P, Li B-Q, et al. A comparison of proliferative capacity and passaging potential between neural stem and progenitor cells in adherent and neurosphere cultures. Int J Dev Neurosci. 2011;29(7):723-31

12. Gilbertson JA, Sen A, Behie LA, Kallos MS. Scaled-up production of mammalian neural precursor cell aggregates in computer-controlled suspension bioreactors. Biotechnol Bioeng. 2006;94(4):783-92.

13. Baghbaderani BA, Behie LA, Sen A, Mukhida K, Hong M, Mendez I. Expansion of human neural precursor cells in large-scale bioreactors for the treatment of neurodegenerative disorders. Biotechnol Prog. 2008;24(4):85970 .

14. Svendsen CN, ter Borg MG, Armstrong RJ, Rosser AE, Chandran S, Ostenfeld $T$, et al. A new method for the rapid and long term growth of human neural precursor cells. J Neurosci Methods. 1998;85(2):141-52.

15. Hansen DV, Rubenstein $J$, Kriegstein AR. Deriving excitatory neurons of the neocortex from pluripotent stem cells. Neuron. 2011;70(4):645-60.

16. Espuny-Camacho I, Michelsen KA, Gall D, Linaro D, Hasche A, Bonnefont J, et al. Pyramidal neurons derived from human pluripotent stem cells integrate efficiently into mouse brain circuits in vivo. Neuron. 2013;77(3): 440-56.

17. Gaspard N, Bouschet T, Hourez R, Dimidschstein J, Naeije G, van den Ameele J, et al. An intrinsic mechanism of corticogenesis from embryonic stem cells. Nature. 2008;455(7211):351-7.

18. Lancaster MA, Renner M, Martin C-A, Wenzel D, Bicknell LS, Hurles ME, et al. Cerebral organoids model human brain development and microcephaly. Nature. 2013:501(7467):373.

19. Sasai Y. Next-generation regenerative medicine: organogenesis from stem cells in 3D culture. Cell Stem Cell. 2013;12(5):520-30.

20. Mariani J, Simonini MV, Palejev D, Tomasini L, Coppola G, Szekely AM, et al. Modeling human cortical development in vitro using induced pluripotent stem cells. Proc Natl Acad Sci. 2012;109(31):12770-5.

21. Rigamonti A, Repetti GG, Sun C, Price FD, Reny DC, Rapino F, et al. Largescale production of mature neurons from human pluripotent stem cells in a three-dimensional suspension culture system. Stem Cell Reports. 2016;6(6): 993-1008.

22. Yan Y, Song L, Madinya J, Ma T, Li Y. Derivation of cortical spheroids from human induced pluripotent stem cells in a suspension bioreactor. Tissue Eng Part A. 2018;24(5-6):418-31.

23. Mcconnell SK. Constructing the cerebral cortex: neurogenesis and fate determination. Neuron. 1995;15(4):761-8.

24. Malatesta P, Hack MA, Hartfuss E, Kettenmann H, Klinkert W, Kirchhoff F, et al. Neuronal or glial progeny : regional differences in radial glia fate. Neuron. 2003:37(5):751-64.

25. Malatesta $P$, Hartfuss $E$, Gotz M. Isolation of radial glial cells by fluorescentactivated cell sorting reveals a neuronal lineage. Development. 2000:127(24): 5253-63. 
26. Zhu X, Ai Z, Hu X, Li T. Efficient generation of corticofugal projection neurons from human embryonic stem cells. Sci Rep. 2016;6:28572.

27. Konno D, Shioi G, Shitamukai A, Mori A, Kiyonari H, Miyata T, et al. Neuroepithelial progenitors undergo LGN-dependent planar divisions to maintain self-renewability during mammalian neurogenesis. Nat Cell Biol. 2008;10(1):93-101.

28. Zhu X, Li B, Ai Z, Xiang Z, Zhang K, Qiu X, et al. A robust single primate neuroepithelial cell clonal expansion system for neural tube development and disease studies. Stem Cell Reports. 2016;6(2):228-42.

29. Li T, Wang S, Xie Y, Lu Y, Zhang X, Wang L, et al. Homologous feeder cells support undifferentiated growth and pluripotency in monkey embryonic stem cells. Stem Cells. 2005;23(8):1192-9.

30. Ai Z, Niu B, Duan K, Si C, Wang S, Xiang $L$, et al. Modulation of Wnt and Activin/Nodal supports efficient derivation, cloning and suspension expansion of human pluripotent stem cells. Biomaterials. 2020;249:120015.

31. Ai Z, Xiang Z, Li Y, Liu G, Wang H, Zheng Y, et al. Conversion of monkey fibroblasts to transplantable telencephalic neuroepithelial stem cells. Biomaterials. 2016;77:53-65.

32. Pertea M, Kim D, Pertea GM, Leek JT, Salzberg SL. Transcript-level expression analysis of RNA-seq experiments with HISAT, StringTie and Ballgown. Nat Protoc. 2016;11(9):1650-67.

33. Stuart T, Butler A, Hoffman P, Hafemeister C, Papalexi E, Mauck WM 3rd, et al. Comprehensive integration of single-cell data. Cell. 2019;177(7):1888902 e21.

34. Lyashenko N, Winter M, Migliorini D, Biechele T, Moon RT, Hartmann C. Differential requirement for the dual functions of $\beta$-catenin in embryonic stem cell self-renewal and germ layer formation. Nat Cell Biol. 2011;13(7):753.

35. Chambers SM, Fasano CA, Papapetrou EP, Tomishima M, Sadelain M, Studer L. Highly efficient neural conversion of human ES and iPS cells by dual inhibition of SMAD signaling. Nat Biotechnol. 2009;27(3):275-80.

36. LaVaute TM, Yoo YD, Pankratz MT, Weick JP, Gerstner JR, Zhang SC. Regulation of neural specification from human embryonic stem cells by BMP and FGF. Stem Cells. 2009;27(8):1741-9.

37. Li W, Sun W, Zhang Y, Wei W, Ambasudhan R, Xia P, et al. Rapid induction and long-term self-renewal of primitive neural precursors from human embryonic stem cells by small molecule inhibitors. Proc Natl Acad Sci U S A. 2011;108(20):8299-304.

38. Sun Y, Pollard S, Conti L, Toselli M, Biella G, Parkin G, et al. Long-term tripotent differentiation capacity of human neural stem (NS) cells in adherent culture. Mol Cell Neurosci. 2008;38(2):245-58.

39. Kan L, Israsena N, Zhang Z, Hu M, Zhao L-R, Jalali A, et al. Sox1 acts through multiple independent pathways to promote neurogenesis. Dev Biol. 2004; 269(2):580-94.

40. Pevny LH, Sockanathan S, Placzek M, Lovell-Badge R. A role for SOX1 in neural determination. Development. 1998;125(10):1967-78.

41. Balzer E, Heine C, Jiang Q, Lee VM, Moss EG. LIN28 alters cell fate succession and acts independently of the let-7 microRNA during neurogliogenesis in vitro. Development. 2010;137(6):891-900.

42. Ruzzo EK, Capo-Chichi J-M, Ben-Zeev B, Chitayat D, Mao H, Pappas AL, et al. Deficiency of asparagine synthetase causes congenital microcephaly and a progressive form of encephalopathy. Neuron. 2013;80(2):429-41.

43. De Castro SC, Malhas A, Leung KY, Gustavsson P, Vaux DJ, Copp AJ, et al. Lamin b1 polymorphism influences morphology of the nuclear envelope, cell cycle progression, and risk of neural tube defects in mice. PLoS Genet. 2012;8(11):e1003059.

44. Zhu H, Junker WM, Finnell RH, Brown S, Shaw GM, Lammer EJ, et al. Lack of association between $\mathrm{ZIC2}$ and $\mathrm{ZIC} 3$ genes and the risk of neural tube defects (NTDs) in Hispanic populations. Am J Med Genet A. 2003;116(4):414-5.

45. Bowman AN, van Amerongen R, Palmer TD, Nusse R. Lineage tracing with Axin2 reveals distinct developmental and adult populations of Wnt/betacatenin-responsive neural stem cells. Proc Natl Acad Sci U S A. 2013;110(18): 7324-9.

46. Wang G, Fulkerson CM, Malek R, Ghassemifar S, Snyder PW, Mendrysa SM. Mutations in Lyar and p53 are synergistically lethal in female mice. Birth Defects Res A Clin Mol Teratol. 2012;94(9):729-37.

47. Kriegstein A, Alvarez-Buylla A. The glial nature of embryonic and adult neural stem cells. Annu Rev Neurosci. 2009;32:149-84.

48. Rash BG, Grove EA. Area and layer patterning in the developing cerebral cortex. Curr Opin Neurobiol. 2006;16(1):25-34.

49. Saito T, Hanai S, Takashima S, Nakagawa E, Okazaki S, Inoue T, et al. Neocortical layer formation of human developing brains and lissencephalies: consideration of layer-specific marker expression. Cereb Cortex. 2011;21(3):588-96.

50. Tsui D, Vessey JP, Tomita H, Kaplan DR, Miller FD. FoxP2 regulates neurogenesis during embryonic cortical development. J Neurosci. 2013; 33(1):244-58.

51. Azevedo FA, Carvalho LR, Grinberg LT, Farfel JM, Ferretti RE, Leite RE, et al. Equal numbers of neuronal and nonneuronal cells make the human brain an isometrically scaled-up primate brain. J Comp Neurol. 2009;513(5):532-41.

52. Alexson TO, Hitoshi S, Coles BL, Bernstein A, van der Kooy D. Notch signaling is required to maintain all neural stem cell populationsirrespective of spatial or temporal niche. Dev Neurosci. 2006;28(1-2):34-48.

53. Klein C, Butt SJ, Machold RP, Johnson JE, Fishell G. Cerebellum- and forebrain-derived stem cells possess intrinsic regional character. Development. 2005;132(20):4497-508.

54. Sart S, Schneider YJ, Li Y, Agathos SN. Stem cell bioprocess engineering towards CGMP production and clinical applications. Cytotechnology. 2014; 66(5):709-22.

55. Zweigerdt R. Large scale production of stem cells and their derivatives. Advances in biochemical engineering $V$. Biotechnology. 2009;114:201-35.

56. Gritti A, Galli R, Vescovi AL. Clonal analyses and cryopreservation of neural stem cell cultures. Methods Mol Biol. 2008;438:173-84.

57. Tropepe V, Sibilia M, Ciruna BG, Rossant J, Wagner EF, van der Kooy D. Distinct neural stem cells proliferate in response to EGF and FGF in the developing mouse telencephalon. Dev Biol. 1999;208(1):166-88.

58. Smukler SR, Runciman SB, Xu S, van der Kooy D. Embryonic stem cells assume a primitive neural stem cell fate in the absence of extrinsic influences. J Cell Biol. 2006;172(1):79-90.

59. Elkabetz Y, Panagiotakos G, Al Shamy G, Socci ND, Tabar V, Studer L. Human ES cell-derived neural rosettes reveal a functionally distinct early neural stem cell stage. Genes Dev. 2008;22(2):152-65.

60. Koch P, Opitz T, Steinbeck JA, Ladewig J, Brüstle O. A rosette-type, selfrenewing human ES cell-derived neural stem cell with potential for in vitro instruction and synaptic integration. Proc Natl Acad Sci. 2009;106(9):3225-30.

61. Deacon TW, Pakzaban P, Isacson O. The lateral ganglionic eminence is the origin of cells committed to striatal phenotypes: neural transplantation and developmental evidence. Brain Res. 1994;668(null):211-9.

62. Wonders CP, Anderson SA. The origin and specification of cortical interneurons. Nat Rev Neurosci. 2006;7(9):687.

63. Butt SJ, Fuccillo M, Nery S, Noctor S, Kriegstein A, Corbin JG, et al. The temporal and spatial origins of cortical interneurons predict their physiological subtype. Neuron. 2005;48(4):591-604.

64. Baizabal J-M, Furlan-Magaril M, Santa-Olalla J, Covarrubias L. Neural stem cells in development and regenerative medicine. Arch Med Res. 2003;34(6): 572-88.

\section{Publisher's Note}

Springer Nature remains neutral with regard to jurisdictional claims in published maps and institutional affiliations.
Ready to submit your research? Choose BMC and benefit from:
- fast, convenient online submission
- thorough peer review by experienced researchers in your field
- rapid publication on acceptance
- support for research data, including large and complex data types
- gold Open Access which fosters wider collaboration and increased citations
- maximum visibility for your research: over $100 \mathrm{M}$ website views per year
At BMC, research is always in progress.
Learn more biomedcentral.com/submissions 